\title{
Global AIDS: A Status Report
}

\author{
by Dr. Jonathan Mann* MD MPH
}

It is amazing and humbling to realize that in the late $1970 \mathrm{~s}$, the human immunodeficiency virus (HIV) was spreading silently - unrecognized and unnoticed - around the world ; - that by 1981, when AIDS was first recognized, cases had already occurred on several continents - and that the worldwide scope of HIV infection and AIDS was not fully realized until the mid-1980s. Yet, while HIV infection and AIDS had stolen a march upon us, the global response has been rapid - the Global AIDS Strategy was designed and adopted - and in the past year we have witnessed an extraordinary and unprecedented global mobilization to prevent and control the disease.

\section{A global problem}

To describe global AIDS, it has been useful to divide the problem into three separate yet inter-dependent epidemics: of infection with HIV; of the disease AIDS; and of the reaction and response - social, cultural, economic, and political - to the HIV and AIDS epidemics. This analysis permits us to focus separately on each element and also emphasizes that the third epidemic of reaction is as much a part of the pathology of AIDS as the virus itself.

On the basis of available information, we believe that HIV is an old if not ancient virus, of unknown geographical origin. The recent history - the current epidemic of HIV infection, the pandemic of global scope, appears to have started in the mid-1970s. From the mid1970 s to the present, we believe that several million people have become infected with HIV. Today, based on available information, we estimate that between 5 and 10 million persons are infected with HIV worldwide. In order to become more precise in our estimation, more valid HIV prevalence data at the national level will be needed. Here we face a very difficult problem - for it has not yet been possible to determine the number of HIVinfected people in any individual country. This task is urgent, for these national estimates provide the scientific basis for predicting the number of AIDS cases which can be expected during the next several years, for targeting prevention programmes, and for determining whether, where and to what extent HIV infection is increasing in the population - which also allows us to measure the effectiveness of prevention efforts.

* Director, Global Programme on AIDS, World Health Organization, Geneva. This paper has been presented to the $15^{\text {th }}$ General Assembly of the Geneva Association, The Hague, 13-14 June 1988. 
Throughout the world, HIV is transmitted in the same basic ways: through sex, blood and from infected mother-to-child. While variations exist regarding, for example, the dominant mode of sexual spread (heterosexual or homosexual) or the principal route of blood transmission (sharing of needles among intravenous drug users or reuse of contaminated needles for medical injections), the fundamental unity of HIV transmission must be emphasized. In addition, despite intense international scientific scrutiny, no evidence has emerged to suggest any change in these modes of HIV transmission. Finally, there is no evidence to support any inherent racial or ethnic resistance to HIV infection or to the pathogenic effects of the virus.

The transformation of the first epidemic - of HIV infection - into the second epidemic of AIDS - is also becoming clearer. Our knowledge necessarily remains limited to the brief period - the decade or less - during which the health status of HIV-infected persons can have been observed. Studies of HIV-infected people have consistently shown that the risk of developing the disease AIDS increases with the length of time they are infected. Expressed in cumulative terms, in the first five years after infection, between 10 and $30 \%$ of HIV-infected people develop AIDS; in some studies, nearly $40 \%$ will develop AIDS by 7 to 8 years after infection. In addition to AIDS itself, other HIV-related illnesses may occur, including persistent generalized lymphadenopathy, AIDS-related complex (ARC) and neurological disease. Thus, in the first five years after infection with HIV, AIDS-related illnesses may occur in $25-50 \%$ of persons, in addition to those who develop AIDS. We do not know if all those infected will ultimately develop AIDS - only the passage of time will clarify the final and complete outcome of HIV infection.

The long delay - of years and perhaps of decades - between HIV infection and disease is critical: thus, essentially all AIDS cases occurring in 1988 and nearly all AIDS cases in 1989 will come from people already infected today and probably several years ago.

As of 10 May 1988, a total of 91905 AIDS cases were officially reported to WHO* from 138 countries around the world. Of all reported cases, $74 \%(68338)$ are from 42 countries in the Americas - 87\% from the United States of America, 12\% (10992) are from 43 African countries, $13 \%$ (11445) are from 28 European countries, and the remaining $1 \%(1065)$ are from 26 countries in Asia and Oceania. Fifty countries have each reported more than 50 AIDS cases to WHO, including 18 countries from the Americas, 14 from Europe, 15 from Africa and 3 from Asia and Oceania.

The number of AIDS cases reported to WHO continues to rise rapidly. In the past four years, the cumulative number of AIDS cases reported to WHO increased over 15 fold. Nearly 100 more countries report AIDS cases today than four years ago. This not only illustrates the widespread awareness of AIDS but also testifies to growing openness and international cooperation. When the rate of increase, rather than the absolute number of reported cases is examined, it is interesting to note that while the time when the curve starts in each continent differs, the slope of the curve is quite similar.

Under the best of circumstances, as many as $90 \%$ of AIDS cases actually occurring in a country will be reported to the national health authority. However, in many areas, the number of reported cases substantially underestimates the actual number of cases, due to

* As of 1 July 1988, a total of 100,410 AIDS cases were reported to the World Health Organization from 138 countries. 
problems in recognizing or diagnosing AIDS, or in reporting AIDS cases to national authorities. Taking these factors into account, we have estimated that since the beginning of the AIDS pandemic a little over a decade ago, the actual number of AIDS cases which have occurred worldwide is approximately 150000.

The epidemic is worldwide, yet the current stage of the HIV epidemic is not the same everywhere and three distinct epidemiological patterns can be described.

The first pattern - Pattern I - involves Western Europe, North America, some areas in South America and Australia and New Zealand. In Pattern I, homosexual and bisexual men and intravenous drug users are the major affected groups. Sexual transmission is predominantly homosexual; over $50 \%$ of homosexual men in some urban areas are HIV infected. While heterosexual transmission is occurring and is increasing in these areas, it currently accounts for a much smaller portion of sexually acquired HIV infections than homosexual transmission. In Pattern I areas, transmission through blood principally involves intravenous drug users. After homosexual and bisexual men, intravenous drug use accounts for the next largest proportion of HIV infections, although in some countries, the majority of AIDS cases occur among intravenous drug users. HIV transmission from blood or blood products is not a continuing problem, as blood for transfusion is screened and blood products are further treated to prevent HIV contamination. In Pattern I areas, most HIV infections have occurred among men; however, perinatal transmission has been documented, primarily among intravenous drug-using women, sex partners of intravenous drugusing men, and among women from pattern II areas of the world.

Pattern II areas include parts of Africa - principally Central, Eastern and Southern and parts of the Caribbean. In Pattern II areas, sexual transmission is predominantly heterosexual and therefore the sex ratio for AIDS cases is approximately equal. In some urban areas, up to $25 \%$ of the $20-40$ year age group may be HIV-infected, although substantial variation has been observed, especially between urban and rural areas. Further, up to 75 to $90 \%$ of female prostitutes in some Pattern II areas may be HIV-infected. In Pattern II, transfusion of HIV-infected blood remains a public health problem. Non-sterile needles, syringes, and other skin-piercing instruments undoubtedly play a role in HIV transmission, but their contribution to the overall burden of HIV infection is smaller than the contribution of sexual transmission. Finally, as a reflection of heterosexual spread, perinatal transmission is a substantial problem; in some areas, 5 to $15 \%$ or more of pregnant women are HIV-infected.

Pattern III areas include Asia, most of the Pacific Region, the Middle East and Eastern Europe. In these areas, HIV seems to have appeared more recently, in the early to mid 1980s. Most AIDS cases in Pattern III areas involve homosexual or heterosexual contact or receipt of imported blood or blood products. In Pattern III areas, the prevalence of HIV infection in high-risk behaviour groups, such as male or female prostitutes, is very low. While HIV infection has not yet penetrated into the general population of Pattern III countries, the virus is present in these areas and evidence of within-country HIV transmission is increasing.

Therefore, while the modes of HIV transmission are identical, the detailed epidemiology of HIV and AIDS differs substantially around the world. Of course, the description of three patterns requires generalization, for different epidemiological patterns may co-exist 
within a country, or even within a large city. Also, the three patterns are not immutable. Over time, in the absence of effective prevention strategies, the three patterns might be expected to blend together. Thus, as safe blood and blood products and safe injections and other skin-piercing practices become the rule worldwide, most HIV transmission will be sexual and perinatal. In addition, regardless of which sexual group is most affected with HIV in a given area today, sexual transmission may ultimately reach all groups who engage in risk behaviour.

The epidemiology of HIV infection also shapes the health impact of AIDS in each society. In general, AIDS predominantly affects two age groups: 20-40 year old adults and infants and very young children.

The impact of AIDS on young adults may be severe in both industrialized and developing countries. Throughout the world, $75-90 \%$ of HIV infections and AIDS cases are occurring among 20-40 year olds. As a result, by 1991, in a Pattern I country, the mortality rate among 25-34 year old men will increase by two-thirds due to AIDS. By 1991, in that same country, the number of deaths from AIDS among men 25-34 years old will be greater than the total number of deaths now occurring in this group from the four current major causes of death - traffic accidents, suicides, heart disease and cancer.

In Pattern II countries, a scenario can be used to describe the health impact of HIV among adults. Thus, in a city of 1 million inhabitants, if $10 \%$ of the $20-50$ year olds are HIV-infected, and assumming that most have been infected fairly recently, AIDS deaths this year would raise the overall adult mortality rate by one-third. By 1991, even assuming that no additional people become infected, the expected AIDS deaths will raise the adult mortality rate by over $100 \%$.

Infants represent another vulverable population, due principally to transmission from infected mothers. In areas where $5 \%$ of pregnant women are HIV-infected, the increase in infant mortality rate due to HIV would be approximately 13 per 1000 , which is greater than the total infant mortality rate, from all causes, in many industrialized countries. In populations with higher levels of infection among pregnant women, such as $20 \%$, increases in infant mortality of 50 per 1000 or more may be anticipated. Despite public health strategies to lower infant mortality, HIV-infection rates of $5-10$ or $20 \%$ among pregnant women will cause infant mortality to rise. Thus, the projected gains in infant and child health from public health efforts, including the Child Survival Initiatives, may be tragically cancelled by AIDS.

The concentrated impact of HIV and AIDS in young adults and infants will cause a decline in national life expectancies in many industrialized and developing countries.

Yet the effects of AIDS go far beyond the health statistics. The third epidemic relentlessly follows the first two epidemics. This is the epidemic of economic, social, cultural and political reaction to HIV infection and AIDS. This worldwide epidemic has only started, yet it is an integral part of the global AIDS problem.

The societal impact of AIDS is linked to the selective loss of persons in the highly productive - in social and economic terms - years of life. In many cultures, 20-40 year old men and women not only provide economic support to children, but also to older persons for whom the family may be the only form of social security. Thus, AIDS is a particularly dangerous threat to family life - young orphans and the elderly will be left behind without support. 
Fear and ignorance continue to lead to tragedies: for individuals, families and entire societies. Unfortunately, as anxiety and fear cause some to blame others, AIDS has unveiled thinly disguised prejudices about race, religion, social class, sex and nationality. As a result, AIDS now threatens free travel between countries and open international communication and exchange.

Further, in the process of fighting AIDS, we find ourselves re-examining many different aspects of health and social systems. Like a diagnostic test, AIDS has helped us to see the inadequacies and inequities in health systems. For to confront AIDS, we must look again and perhaps differently at social problems like prostitution of women, children and men or intravenous drug use; we must recognize long-standing weaknesses in health care systems regarding blood, needles and invasive practices; and we find ourselves reexamining the ways we speak to, inform and educate each other about health. AIDS remorselessly highlights our most complex problems, challenges our assumptions and shakes our complacency.

In seeking now to project into the future, we are limited by our incomplete knowledge. Nevertheless, using available data we estimate that approximately 150000 new cases of AIDS will occur during this year. Therefore, the number of AIDS cases during 1988 will equal the total number of cases which have thusfar occurred worldwide. If we adopt the conservative estimate that 5 million people are infected today with HIV, a cumulative total of one million AIDS cases would be expected by 1991. Thus, the period 1988-91 will likely witness over 5 times more AIDS cases than have thusfar occurred.

What then, is the potential for spread of HIV? Studies of intravenous drug users, homosexual and bisexual men, and women prostitutes have shown clearly that if the virus is present in the community and the behaviours that transmit infection are sufficiently common and intense, HIV has the capacity to create explosive epidemics.

In addition, HIV infection is lifelong, so the virus can survive in the human population if, during the lifetime of an infected person, it can spread to one other person. This suggests that HIV infection will relatively easily perpetuate itself - unless a curative treatment or a preventive vaccine is developed - and neither is likely in the next several years. Thus, HIV infection is likely to remain with us for the foreseeable future.

The full impact of HIV will be felt over a period of decades. HIV does not need to spread rapidly in a population to have a tremendous and gradually expanding cumulative effect. The two major factors influencing the risk of individual infection are the prevalence of HIV in the community and the individual's personal behaviour. We do not have precise numbers, but it is likely that several hundred million people around the world may have behaviours which make them potentially vulnerable to infection with HIV. Thus, while it has become fashionable to reassure and state that AIDS will never threaten large populations, we believe that virology, immunology, sociology and epidemiology require us to take the long view - and a more somber view. Let us remember that we are still in the early phases of a gobal epidemic whose first decade gives us every rational reason for concern about the global future of AIDS. 


\section{A global response}

A global problem - a global response.

The World Health Organization has the constitutional responsibility to direct and coordinate international health work.

In May 1986, the World Health Assembly supported an intensification of the AIDS Programme within WHO. The Director-General, Dr. Mahler, reviewed the data collected by WHO and concluded that the world was facing a global emergency requiring urgent action.

On 20 November 1986, Dr. Mahler went to the United Nations Headquarters in New York where he gave a worldwide press briefing on this new global health priority. His stark assessment: "we stand nakedly in front of a pandemic as mortal as any pandemic there has ever been".

Immediate action was taken within WHO to reinforce the capability of the AIDS programme. An intensive reformulation of programme goals, objectives and needs was undertaken. By January 1987 the Global AIDS Strategy had been finalized and distributed and on 1 February 1987, the Special Programme on AIDS (SPA) was established. The two major tasks facing WHO were to support national AIDS prevention and control programmes around the world, and to provide global leadership and help ensure international collaboration and cooperation.

In May 1987 the Fortieth World Health Assembly endorsed the global strategy for the prevention and control of AIDS, and the establishment of the Special Programme on AIDS as the vehicle for its implementation. The Global AIDS Strategy has been further unanimously approved and adopted as the foundation for global action by the Venice Summit (June 1987), the Economic and Social Council of the United Nations (ECOSOC - July 1987), the United Nations General Asembly (October 1987) and the World Summit of Health Ministers in London (January 1988).

In January 1988, the Special Programme was renamed the Global Programme on AIDS.

The Global AIDS Strategy establishes basic principles for national and international AIDS prevention and control, based firmly upon knowledge of HIV virology and epidemiology, and derived from broad and practical experience with programmes to control infectious diseases. Therefore, the Global AIDS Strategy provides the necessary framework, within which each country must develop its own detailed strategy.

The Global AIDS Strategy has three objectives:

- to prevent HIV infection;

- to reduce the personal and social impact of HIV infection, and to care for those already infected with HIV, and with AIDS;

- to unify national and international efforts.

The Global Strategy is based on the following principles:

- public health must be protected;

- human rights must be respected and discrimination must be prevented; 
- we know enough now to prevent the spread of HIV, even though a vaccine is not yet available;

- education is the key to AIDS prevention precisely because HIV transmission can be prevented through informed and responsible behaviour;

- AIDS control will require a sustained social and political commitment;

- all countries need a comprehensive national AIDS programme, integrated into national health systems and linked within a global network;

- systematic monitoring and evaluation will ensure that the Global Strategy can adapt and strenghten.

In addition to marshalling the support of all nations, WHO has mobilized the human and financial resources to begin the rapid implementation of this Strategy around the world. The resulting effort has replaced the chaotic anxiety about AIDS which was typical of 1985 with the structured, purposeful and increasingly powerful work of current national and international AIDS prevention and control activity.

WHO support to national AIDS programmes has reached an unprecedented level with 136 countries having entered into collaboration with the Global Programme on AIDS. This support is designed to support each country to develop, implement and evaluate its own national AIDS prevention and control programme, in conformity with the Global AIDS Strategy. Over 150 countries have now established National AIDS Committees.

WHO has completed over 300 consultant missions to a total of 115 countries. A further 21 countries will be visited by the end of the third quarter of 1988 . Of these countries, 44 are African, 38 American, 6 European, 8 Middle Eastern, and 30 in Asia and Oceania.

WHO missions have resulted in the preparation of 80 short-term (6-12 month) and 22 medium-term (3-5 year) national AIDS control plans. A further 31 comprehensive medium-term plans are nearing completion.

Following the official endorsement of the national medium-term plan, national donor meetings have been jointly organised by the Ministry of Health and WHO in 8 Member States: Uganda (May 1987), United Republic of Tanzania (July 1987), Rwanda (July 1987), Kenya (July 1987), Ethiopia (August 1987), Zaire (February 1988), Senegal (February 1988) and Zambia (March 1988).

A total of US $\$ 35$ million was pledged at these meetings, with the funds to be made available either through WHO/GPA or bilaterally within the framework of the approved national AIDS plan.

\section{The Global AIDS Strategy objectives}

The first objective of the Global AIDS Strategy, preventing HIV transmission, is achievable precisely because HIV is transmitted through specific individual behaviours and through readily indentified practices in the health system. 
Individual behaviour is responsible for most HIV transmission. This transmission requires the active participation of two persons; therefore, the chain of transmission can be broken by the individual behaviour of either the infected or the non-infected person. For this reason, the proper focus of prevention is behaviour, not infection status.

To prevent HIV transmission, we must inform and educate, to prevent the adoption of risk behaviours, or to help people with such behaviours to abandon or modify them. For this task, a four-part information and education programme will be needed: for the general public, for target groups in the population, for specific individuals and for health workers.

Programmes for the general public alert and inform about AIDS; for these programmes to succeed, the media must be well informed and supportive. Yet the behaviours involved in AIDS transmission are private, hidden from others, or frankly disapproved by many societies. Since we cannot know everyone who already has or may develop risk behaviours, everyone should be given information and education about AIDS. These programmes also establish AIDS as a legitimate issue - an issue on the national agenda, worthy of discussion and action. Yet, these broad public information campaigns do not and should not be expected to produce rapid or sustained behaviour change. The need to speak to an entire population - young and old, rich and poor, women and men, educated and uneducated - inevitably makes of broad public programmes a blunt instrument.

Not everyone has an equal risk of HIV infection. Information and education, like any health intervention, should be targeted to where it is most needed. So, we need to know more about the private behaviours of our peoples - and while this can be done, it must be done carefully, with empathy and sensitivity - or vital information will be hidden and nothing useful will be learned.

Information and education for target groups must involve their active participation in all phases of the programme - planning, implementing and evaluating. The alternative to working with the people you wish to educate is dismal failure. For if people believe that AIDS is a curable disease they will not use condoms. If intravenous drug users do not understand the words you use to describe needles, syringes and other drug equipment, they cannot learn prevention. If adolescents are convinced that AIDS is being used to deprive them of sexual freedom, they may refuse to listen to prevention messages and even counterreact in ways that further endanger their lives. There is a universal lessen - that "we" - whoever we are and regardless of our experience - do not know best how to educate "them". We need an alliance - a dialogue not a monologue - and there are many examples throughout the world to show that when people from target groups are asked to become involved in AIDS information and education programmes, they respond positively, sincerely - and often with great energy and creativity.

Yet some individuals will need even more, and an even more personal contact - not only to inform and educate, but also to support the difficult process of behaviour change. Counselling - a key component of information and education programmes - must be organized and provided to individuals and small groups. Counselling is a more personal and intimate realm in which the person with high-risk behaviour, the person seeking voluntary HIV testing, the HIV-infected person, their families and friends, can find information, understanding and support. 
The fourth information and education component is directed to health workers who must not only meet the challenges of informing and educating others, providing humane care to infected persons and ensuring the safety of practices in the health system but who must contribute actively - through personal and community leadership - to an enlightened public opinion.

These are the four components of national information and education programmes; yet by themselves, such programmes are not enough. Prevention cannot live by information alone - information and education programmes can succeed only if there is a supportive social environment and if certain health and social services are made available.

A supportive social environment includes tolerance and avoiding discrimination. Those who are infected, or who need help to stop risk behaviour, are also part of our society and part of us. There is no public health rationale to justify isolation, quarantine, or other discriminatory measures based solely on a person's HIV infection status or practice of risk behaviours. Preventing discrimination not only protects human rights, but helps ensure an effective AIDS programme. Discrimination and fear will undermine the entire national information and education programme; thus, discrimination itself can endanger public health.

Certain health and social services are also needed to support and strengthen people's capacity to make long-term behaviour changes. Examples of these services include: treatment programmes for intravenous drug users so they may stop abusing drugs; long-term counselling services for infected persons, their sexual partners and their families; voluntary HIV testing services; and condoms. Good intentions are not enough. How realistic is HIV control among intravenous drug users if treatment programmes for drug abuse are not available? How likely is long-term behaviour change without long-term access to counselling, support and advice? How likely is condom use if condoms are too expensive, of poor quality, or simply not available?

Thus, national efforts to prevent HIV transmission through behaviour include a fourpart information and education programme, a supportive social environment and needed health and social services. Each is essential; lack of any component will dramatically reduce the national prevention capacity.

The second objective of the Global AIDS Strategy is to reduce the personal and social impact of HIV infection. This means providing humane care, of a quality not inferior to that for other diseases, to those ill with HIV, and providing counselling, social support and services to those infected but not ill. Reducing the social impact also requires broad social and political commitment, the strength to reject simplistic solutions for AIDS control, and the will to ensure participation of the entire health and social sector in an active programme "Against AIDS - For Health".

The third objective of the Global AIDS Strategy is to unify national and international efforts against AIDS. 1987 was the year of AIDS mobilization - at the national and global levels. There is simply no parallel to what was accomplished in 1987.

In addition to supporting national AIDS plan development, implementation and evaluation, the WHO Global Programme on AIDS has: 
- ensured that the broad social, cultural, economic and political impacts of HIV infection and AIDS are recognized by national and international organizations, including all components of the United Nations family;

- facilitated and promoted biomedical, social, behavioural and epidemiological research on HIV infection and AIDS;

- ensured a national and international commitment to information, to developing needed information and for the free and open exchange of this vital information;

- developed guidelines regarding a series of complex and difficult policy issues, such as: HIV and international travel; social aspects of national AIDS programmes; AIDS and prisons; HIV infection and breast-feeding; neuropsychiatric aspects of HIV infection; and screening and testing policies;

- emphasized the commitment to human rights as an integral part of the fight against AIDS.

WHO has taken the lead to issue policy statements regarding the vital need to protect human rights in the fight against AIDS. WHO believes that human rights are not only vital to protect for their own sake, but that discrimination and other violations of the human rights of HIV-infected persons will also diminish the efficiency and effectiveness of national AIDS prevention programmes. Therefore, protection of human rights is also a public health priority. Accordingly, WHO is organizing a series of meetings of agencies (UN and NGO) with a major role in the human rights field, to inform these groups about the technical issues involved, about the policies already developed by WHO on social aspects of HIV control, screening for HIV infection, international travel, and AIDS in prisons, and to develop a common strategy to ensure protection of human rights in the fight against AIDS.

WHO is setting up a Global Commission on AIDS to provide it with expert advice from eminent persons from a wide variety of disciplines with applicability to the Global Programme on AIDS. This Commission will give advice on short, medium and long-term priorities in the scientific and technical components of the Global Programme, including the establishment of scientific working groups.

WHO has been working closely with many parts of the UN family to encourage and support increasingly active participation in AIDS control activities. WHO has entered an alliance with UNDP to coordinate efforts at the country level. WHO collaboration on AIDS with organizations of the UN system is intensifying - with UNESCO on AIDS education in school, UNICEF - mothers and children, UNFPA - family planning, ILO - policy and education in the workplace, FAO - impact on food production, World Bank - economic and demographic impact, and WTO on information for travellers.

The Global Programme on AIDS has now received pledges and funding from 16 countries (in alphabetical order): Australia, Belgium, Canada, Denmark, Finland, France, Italy, Japan, the Netherlands, New Zealand, Norway, Sweden, Switzerland, the Union of Soviet Socialist Republics, the United Kingdom, and the United States. In addition, funding has been received from the United Nations Development Programm (UNDP) and the Sasakawa Medical Foundation. 
Information and education were the focus of the World Summit of Ministers of Health on Programmes for AIDS Prevention which was jointly organized by WHO and the Government of the United Kingdom in London from 26 to 28 January 1988. It was a truly historic landmark not only for AIDS prevention and control but also for health. Never before had such a large number of Ministers of Health met, evidencing an unprecedented political commitment to a health issue. The 148 national delegations comprised 114 Ministers who remained throughout the three-day Summit - Against AIDS, For Health.

World AIDS Day, scheduled for 1 December 1988, has as its theme "tell the world what you're doing about AIDS". WHO is coordinating the Day's activities, during which national governments - and all organizations and institutions working on any aspect of AIDS research, prevention, control and treatment - will be encouraged to explain to their communities what they are doing about AIDS. We look to this day as a time when people throughout the world, working as individuals and together, will see how their efforts are linked in the global struggle against AIDS and feel united in the global community.

The Global AIDS Strategy, fully applied in every country, will start the sequence that leads - through behaviour change - to begin to slow the spread of the AIDS virus itself. Thus, in this year - 1988-it is possible to enter a new era in our confrontation with AIDS.

As the Summit declaration concluded:

"We are convinced that, by promoting responsible behaviour and through international cooperation, we can and will begin now to slow the spread of HIV infection." 